\title{
Retrieving Common Discretionary Lane Changing Characteristics from Trajectories
}

\author{
Li Li, Fellow, IEEE, Chen Lv, Member, IEEE, Dongpu Cao Member, IEEE, and Jiajie Zhang
}

\begin{abstract}
Conventional lane change methods directly collected steering angle data via onboard sensors to accurately capture the actions of individual drivers. We can hardly use such methods to collect massive data from examinees, because of time and financial costs. In order to retrieve common steering behaviors for lots of drivers, we propose a method to retrieve common Discretionary Lane Change (DLC) steering characteristics from trajectory data. The key technique of this new method is solving an inverse problem that converts the measured trajectory into the unmeasured steering maneuvers under the assumed vehicle movement dynamics. We find that most normal DLC trajectories in the Next Generation Simulation (NGSIM) datasets could be well reproduced by a simple target heading angle preview control model. This finding sheds important light into driver behavior study and better explains how human control vehicles. Based on these findings, we can non-intrusively evaluate driving performance or physiological states of drivers based on online roadside monitoring data (e.g. the data collected from roadside video cameras). This opens a promising field of applications for enhancing driving safety.
\end{abstract}

Index Terms - Lane change, steering, target heading angle model

\section{INTRODUCTION}

D RIVING behaviors attract attentions from investigators of traffic safety [1], developers of advanced driver-assistance systems (ADASs) [2]-[3], designers of microscopic simulation software [4]-[6] and researchers in many other fields. Recent studies had proven that it is important to integrate driving behavior models into ADASs design to reduce drivers' burden and also enhance driving safety [7].

In this paper, we focus on a subtopic within this field: lane changing behaviors modeling [8]. As well known, there are two types of lane changes: Mandatory Lane Changes (MLC) occurs when drivers must change lane to follow their paths; and Discretionary Lane Changes (DLC) occurs when drivers

Manuscript received on September 10, 2017. This work was supported in part by the National Natural Science Foundation of China (Grant No. 91520301) and the Beijing Municipal Science and Technology Project (No.D171100000317002). (Corresponding author: Dongpu Cao)

$\mathrm{L}$. $\mathrm{Li}$ is with Department of Automation, Tsinghua National Laboratory for Information Science and Technology (TNList), Tsinghua University, Beijing, China 100084. (Email: li-li@ @tsinghua.edu.cn)

C. Lv and D. Cao are with Centre for Advanced Vehicle Engineering, Cranfield University, Cranfield, MK43 0AL, UK. (Email: c.lyu@cranfield.ac.uk, d.cao@cranfield.ac.uk)

J. Zhang is with Alibaba Cop.. change lane to improve navigation speeds. A variety of lane change models were then proposed to describe these two kinds of lane changes. Most of these models emphasize decision process of lane change [9]-[12]. Differently, in this paper, we will address drivers' steering maneuvers and associate visual attentions. To concentrate on human steering features, we will focus on DLC in this paper.

In general, we have two kinds of approaches to describe DLC maneuvers. The first kind of approaches uses onboard meters to record a DLC maneuver as a time series of steering angles [13]-[15]. Such approaches capture accurate information of individual drivers. However, it is hard to retrieve common features of DLC maneuvers from many candidates via this kind of approaches, since it requires significant financial and time costs to collect massive field test data from lots of examinees. Moreover, whether one driver's behavior remains unchanged within urban streets and proving grounds needs to be further validated [16].

The second kind of approaches uses a time series of vehicle trajectory to describe a DLC maneuver [17]-[19]. With quick development of computer vision and machine learning skills, video-based vehicle trajectory monitoring technologies become pervasive in practice and provide a huge amount of data to support driver behavior study [20]-[21]. Vehicle trajectories contain rich information of individual drivers and allow us to study interactions between drivers or even monitor behaviors of drivers online.

It had been shown that most observed DLC trajectories have a similar "S"-shape. In [19], it had been further demonstrated via Next Generation Simulation (NGSIM) dataset [22] that the kernel part of a normal DLC trajectory can be approximately depicted by a certain 5th-order polynomial and the durations of DLC maneuvers follow a speed-dependent log-normal distribution. NGSIM is a public-open dataset consist of detailed vehicle trajectory, wide-area detector, and supporting data for researching driver behavior. Its high-resolution trajectory data had been used in a number of traffic flow and driving behavior studies.

Although we can use these DLC trajectory models to notably improve both temporal and spatial accuracy of microscopic traffic simulation models, we still need to further explain what physiological characteristics of drivers lead to these "S"-shape trajectories. In other words, we aim to integrate steering type and trajectory type studies on DLC maneuvers.

One potential way to reach this goal is the global trajectoryoriented steering control models. Such models assume that a 
driver will first generate in his/her mind the whole "S"-shape DLC trajectories that mostly fits empirical ones [23]-[26] and then implement a steering control to guarantee vehicles track the planned trajectories [27]-[31]. However, these models artificially separate DLC maneuvers into two parts: trajectory generation/planning and trajectory tracking. This separation lacks of psychology supports.

Another way to integrate these two kinds of studies is the local deviation-oriented steering control models. Such models often assume a driver evaluates his/her deviation to the desired DLC state from time to time and apply a steering control to reduce the estimated deviation. The "S"-shape DLC trajectory will be then generated naturally but not intentionally [32]-[36].

Such models are called preview/prediction model, too. The term preview means that a human driver will perceive future path information within a finite future distance through visual cues. The term prediction means that a human driver will establish a vehicle dynamic model in his/her mind and predict vehicle's future trajectories.

However, such approaches usually calibrate and validate their models according to the recorded steering angle time series. As a result, the common DLC characteristics of many examinees are left unsolved due to costs limits.

Recently, yet an alternative way to integrate both kinds of studies was proposed in [37], where we need both steering angle data and the corresponding trajectory. In current market vehicles, the steering angle can be measured by on-board sensors. However, the heading angle and the real-time position need additional high-resolution GPS sensors. We cannot install such sensors on thousands of vehicles, because of the time and financial costs. So, we directly estimate steering maneuvers from empirical DLC trajectories. However, the estimation algorithm proposed in [37] is time consuming and is also sensitive to measurement errors. This prevents us retrieving the common features of drivers from massive trajectory data. Nevertheless, the psychological origins of the steering control strategy were not analyzed in [37].

In this paper, we propose a new method to retrieve common DLC behaviors from massive vehicle trajectories retrieved from video data. As a result, we can non-intrusively evaluate driving performance or physiological states of drivers. As shown in [7], when on-board video sensors are unavailable, we often resort to indirect measurements as an alternative to monitor driving actions. Following this idea, we can establish a detection model for abnormal lane changing behaviors based on position measurement of vehicles. If the estimated heading angle changes too abruptly during lane change for multiple times, we may concern whether the driver is in abnormal state or his/her driving performance is good enough to run on road. This opens a promising field of applications for enhancing driving safety.

Based on the estimated steering angle time series, we find that most DLC trajectories recorded in NGSIM datasets could be reproduced by a simple target heading angle model. This finding sheds important light into DLC behavior study and better explains how human drivers control vehicles.

To present the details, the rest of this paper is arranged as follows: Section II reviews the bicycle model of steering maneuvers and then discusses how to use it to estimate driving maneuvers from vehicle trajectory data. Section III studies a target heading angle model to explain drivers' control strategy during DLC. Estimating the steering maneuvers of the sampled trajectories from NGSIM dataset, Section IV discusses the common features of DLC actions based on the proposed target heading angle model. Fig. 1 gives the flowchart of all the issues studied in this paper. Finally, Section $V$ concludes the findings and discusses the possible applications of the proposed model.

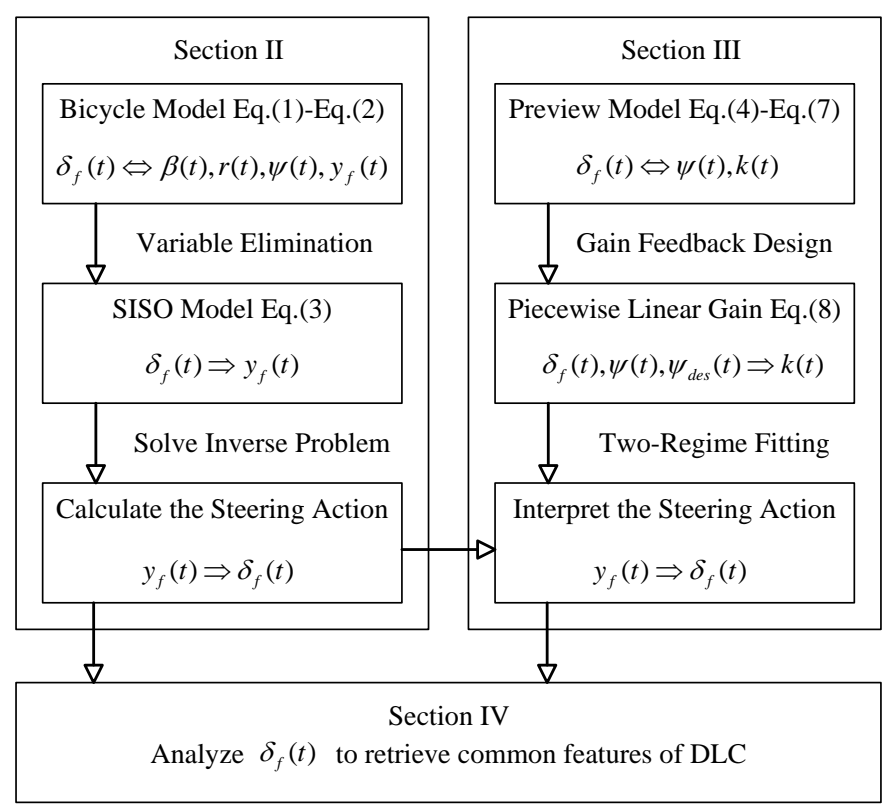

Fig.1. The flowchart of all the issues studied this paper. The meanings of the symbols in this figure will be explained in the below.

\section{EstimATING STEERING MANEUVERS FROM TRAJECTORIES}

\section{A. The Bicycle Model}

The key technique of our estimation method is solving an inverse problem that converts the measured trajectory into the unmeasured steering maneuvers under the assumed vehicle movement dynamics. Therefore, we will briefly introduce the vehicle model in this subsection.

Since most vehicles recorded in NGSIM datasets are front steering vehicles, we choose the frequently applied Bicycle model (shown in Fig.2) to describe vehicle lateral mechanics. Bicycle model may be inaccurate, if the steering angle is too large. However, for Discretionary Lane Change (DLC) actions, the steering angle usually remains so small that the error can be tolerated. As shown in [38]-[39], the Bicycle model is accurate enough for our study.

It should be pointed out that we could apply certain nonlinear lateral motion models when we estimate the steering action. However, we need to further connect the lateral motion model that we used to the preview control model. If a nonlinear kinematic model is used, we cannot directly apply it to the previous control model. So, we choose the bicycle model here. Table I lists the corresponding symbols used in this paper. 
In short, the Bicycle model assumes that the steering angle $\delta_{f}(t)$ causes the change of yaw rate $r(t)$, displacement from the front tire to the guideline $y_{f}(t)$ (we used vision sensor to measure the displacement from the front time. The reference point locates at the desired trajectory such that the line segment links the front tire and the reference point is perpendicular to the center line of the vehicle), and heading angle $\psi(t)$.

TABLE I

NOMENCLATURE LIST

\begin{tabular}{ll}
\hline \hline Symbol & \multicolumn{1}{c}{ MEANING } \\
\hline$v(t)$ & Vehicle velocity \\
\hline$\psi(t)$ & $\begin{array}{l}\text { Heading angle from guideline line to longitudinal axis } \\
\text { of vehicle body } \boldsymbol{B} \boldsymbol{B}\end{array}$ \\
\hline$\beta(t)$ & $\begin{array}{l}\text { Slide-slip angle from longitudinal axis of vehicle body } \\
\boldsymbol{A} \boldsymbol{B} \text { to the direction of vehicle velocity }\end{array}$ \\
\hline$r(t)$ & Yaw rate \\
\hline$\delta_{f}(t)$ & Front tires steering angle \\
\hline$f_{f}$ & $\begin{array}{l}\text { Front tire force which is perpendicular to the direction } \\
\text { of front tire movement }\end{array}$ \\
\hline$f_{r}$ & $\begin{array}{l}\text { Rear tire force which is perpendicular to the direction } \\
\text { of rear tire movement }\end{array}$ \\
\hline$y_{f}$ & Displacement from front tire to guideline \\
\hline$m$ & Mass of the vehicle \\
\hline$I_{z}$ & Inertia moment around z-axis \\
\hline$l_{f}$ & Distance from $\boldsymbol{A}$ and $\boldsymbol{C G}$ \\
\hline$l_{r}$ & Distance from $\boldsymbol{B}$ and $\boldsymbol{C G}$ \\
\hline$c_{f}$ & Stiffness coefficient of front tire \\
\hline$c_{r}$ & Stiffness coefficient of rear tire \\
\hline$g$ & Acceleration of gravity force \\
\hline \hline
\end{tabular}

The detailed time-domain system dynamic equation is written as [39]

$$
\left[\begin{array}{c}
\dot{\beta}(t) \\
\dot{r}(t) \\
\dot{\psi}(t) \\
\dot{y}_{f}(t)
\end{array}\right]=\left[\begin{array}{cccc}
a_{11} & a_{12} & 0 & 0 \\
a_{21} & a_{22} & 0 & 0 \\
0 & 1 & 0 & 0 \\
v & l_{f} & v & 0
\end{array}\right]\left[\begin{array}{c}
\beta(t) \\
r(t) \\
\psi(t) \\
y_{f}(t)
\end{array}\right]+\left[\begin{array}{c}
b_{11} \\
b_{21} \\
0 \\
0
\end{array}\right] \delta_{f}(t)
$$

where

$$
\begin{gathered}
a_{11}=-\left(c_{r}+c_{f}\right) / \tilde{m} v, a_{12}=-1+\left(c_{r} l_{r}-c_{f} l_{f}\right) / \tilde{m} v^{2} \\
a_{21}=\left(c_{r} l_{r}-c_{f} l_{f}\right) / \tilde{I}_{z}, a_{22}=-\left(c_{r} l_{r}^{2}+c_{f} l_{f}^{2}\right) / \tilde{I}_{z} v \\
b_{11}=c_{f} / \tilde{m} v, b_{21}=c_{f} l_{f} / \tilde{I}_{z}
\end{gathered}
$$

in which $\tilde{m}=m / \lambda$ and $\tilde{I}_{z}=I_{z} / \lambda$ are the normalized mass and inertia, respectively. We use parameter $\lambda$ to model road adhesion. For dry road, we often set $\lambda=1$; and for wet road, $\lambda=0.5$. Since NGSIM dataset were collected in sunny days, we set $\lambda=1$ in the rest of this paper.

During a lane change, we vary the steering angle $\delta_{f}(t)$ so as to change $y_{f}(t)$ from 0 to a desired value after certain time interval; meanwhile, we need to guarantee $r(t)$ and $\psi(t)$ are approximately 0 at the beginning time and the ending time of lane change.

For simplicity, we neglect the gap between the front bump and the front tire of the vehicle in this paper. So, a trajectory recorded in NGSIM dataset refers to the trajectory of the front tire of the studied vehicle in the follows.

We had also investigated the speed change during DLC manoeuver in reference [17]. Tests showed that drivers usually do not significantly change their speeds during DLC. So, we do not further discuss the speeds on different lanes and assume a constant speed when using Bicycle model.

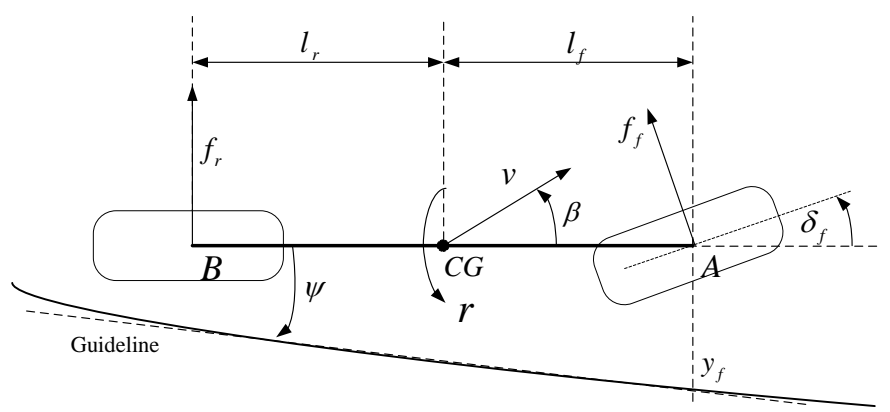

Fig.2. The "Bicycle Model" for front steering vehicles, in which $\boldsymbol{C} \boldsymbol{G}$ represent the center of gravity for vehicle body. Symbol $\boldsymbol{A}$ and $\boldsymbol{B}$ denote the positions of front and rear tire/road interfaces, respectively.

\section{B. The Estimation Algorithm of Steering Maneuvers}

Based on the time-domain model (1), we can eliminate the other variables and derive the time-domain transfer function between $\delta_{f}(t)$ and $y_{f}(t)$ as

$$
d_{0} \delta_{f}+d_{1} \dot{\delta}_{f}+d_{2} \ddot{\delta}_{f}=n_{1} \dot{y}_{f}+n_{2} \ddot{y}_{f}+n_{3} \dddot{y}_{f}+n_{4} y_{f}^{(4)}
$$

where $n_{1}, n_{2}, n_{3}, n_{4}, d_{0}, d_{1}, d_{2}$ are constant coefficients determined from Eq.(1); see Appendix I for detailed expression of these coefficients.

Since we can retrieve $y_{f}(t)$ and all its first-order as well as higher-order derivatives from trajectory data, the right hand side of Eq.(3) will be determined. Then, with given vehicle parameters, we can solve the unmeasured $\delta_{f}(t)$ from a second-order ordinal differential equation (ODE). Noticing that this is a well-posed ODE problem, we can easily reconstruct all the status variables of the vehicle by applying the bicycle model Eq. (1), if $\delta(t)$ is known.

However, there are mainly three difficulties in applying this solving algorithm.

First, raw trajectory data contain some abnormal driving maneuvers [17], [19]; see Fig.3 for an example. So, we may draw biased conclusions, if raw data labeled as DLC cases in NGSIM were directly used without properly examination. To solve this problem, we follow the suggestion given in [19] and use trajectory clustering methods [40]-[41] to automatically filter out abnormal DLC lane change maneuvers from massive sampled data. The details of such filtering can be found in [19] and are thus omitted here.

Second, raw trajectory data are not smooth and contains some measurement errors. We should smooth out these small 
bursts in $y_{f}(t)$ to avoid that large peaks appear in the higher-order derivatives of $y_{f}(t)$.

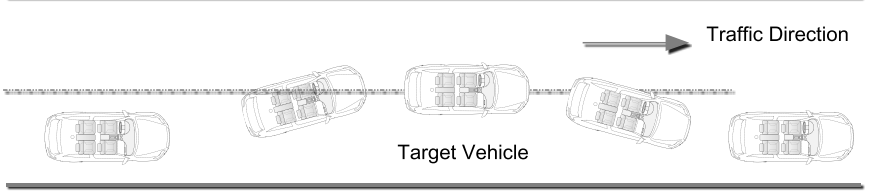

(a)

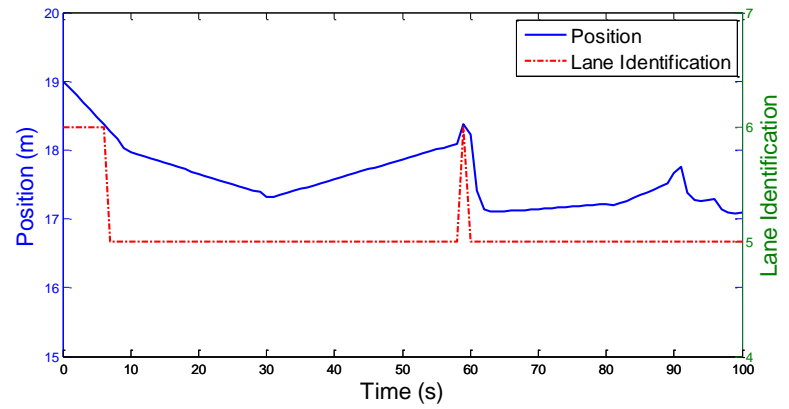

(b)

Fig.3. (a) An general bird-view illustration for the case that a vehicle wanders near the lane boundary; (b) a special case of wandering vehicle found in NGSIM dataset; where the varying process of the vehicles' position is plotted.
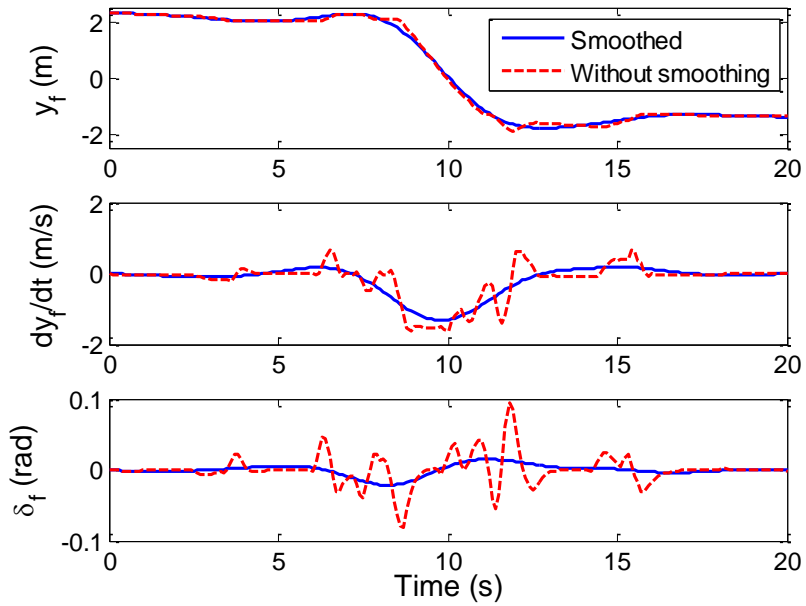

Fig.4. A demonstration on the effect of B-spline smoothing. The chosen trajectory is the no. 383 trajectory. Dashed curves represent the results estimated directly from empirical data without smoothing.

Solid curves represent the results estimated from smoothed data. The parameters of the Bicycle model are chosen as $m=1500 \mathrm{~kg}$, $I_{z}=2500 \mathrm{kgm}^{2}, l_{f}=2.2 \mathrm{~m}, l_{r}=2.2 \mathrm{~m}, c_{f}=50000 \mathrm{~N} / \mathrm{rad}$,

$$
c_{r}=64000 \mathrm{~N} / \mathrm{rad} \text {. }
$$

In this paper, we choose the B-spline approximation method [40] to fit and smooth the trajectory. The term "B-spline" is an abbreviation for basis spline. In short, a spline function is a piecewise polynomial function. The places where these pieces meet are called knots. The number of knots are constrained so that the spline function has limited support. The key property of spline functions is that they are continuous at the knots, so the derivatives of a spline function may also be continuous. Thus,
B-spline curve has good mathematical properties including the continuity and smoothness for certain order derivatives. It guarantee the estimated higher-order derivatives of $y_{f}(t)$ are

robust to measurement noises or small disturbances.

Particularly in this paper, we chose 5th-order B-spline in order to obtain continuous and smooth 4 th-order derivatives of $y_{f}(t)$. Fig.4 demonstrates the estimated steering angle time series with and without B-spline approximation.

Third, the second-order ODE Eq.(3) of $\delta_{f}(t)$ is stiff. We should be careful in choosing the numerical algorithms. Some detailed explanations on stiff ODE and the corresponding numerical algorithms could be found in [43]. We will not further discuss this problem here.

\section{The Trajectory Dataset}

The trajectory data used in this paper are obtained from two locations. The first part of data was collected on U.S. Highway 101 in Los Angeles, CA, during morning rush hour from 7:50 am to 8:35 am on June 15 2005. The second part of data was collected on U.S. Highway, Emeryville, CA, during evening rush hour from 4:00 pm to 4:15 pm and 5:00 pm to 5:30 pm on April 13, 2005. After filtering, we will get 1027 normal DLC trajectories [19].

Following the suggestions given in [19], we only consider the kernel part of each lane changing trajectory with a uniform length (20 seconds) in this paper, because few normal lane changes last longer than 20 seconds [44]-[45]. Suppose the lane change happens at time $T$ (as defined in NGSIM, the time that the center of the vehicle passes the lane boundary), the segment of this trajectory with time range $t \in[T-10 \mathrm{~s}, T+10 \mathrm{~s}]$ will be then studied. This alignment trick prevents obtaining large Euclidean distance between two similar trajectories due to time shifts.

\section{The Influence of the Uncertainties in Vehicle Parameters}

After solving these three difficulties, we can estimate driver steering maneuvers from trajectory with given Bicycle model. However, we cannot determine all parameters of the Bicycle model corresponding to a trajectory directly. Therefore, we need to discuss the influence of the uncertainties in vehicle parameters.

$l_{f}$ and $l_{r}$ are a pair of important parameters. Based on video processing, the vehicle length $l_{f}+l_{r}$ can be obtained directly from raw data of NGSIM. So we assume it is accurate enough. What we do not know is the partition between $l_{f}$ and $l_{r}$.

The remaining uncertainty in vehicle geometry is the location of gravity center. Tests show that, if we vary $l_{f}$ from $1.5 \mathrm{~m}$ to $2.9 \mathrm{~m}$ and meanwhile keep the vehicle length $l_{f}+l_{r}$ fixed, the estimated steering angles times series are roughly the same, with other parameters of the Bicycle model remain unchanged; see Fig.5 for an example. 
The mass and inertia moment of vehicles are another pair of important parameters. For the vehicles in NGSIM dataset, the mass of vehicles should be $1000 \mathrm{~kg} 2500 \mathrm{~kg}$; and the inertia moment will be proportional to the mass. If we vary $m$ from $1000 \mathrm{~kg} 2500 \mathrm{~kg}$ and meanwhile keep the inertia moment proportional to the value of mass, the estimated steering angles times series are almost the same, with other parameters of the Bicycle model remain unchanged; see Fig.6 for an example. This is partly because the change of $m$ and $I_{z}$ will leads to the proportional changes of the coefficients $a_{11}, a_{12}, a_{21}, a_{22}$, $b_{11}, b_{21}$ in Eq.(1). So, the steering angle time series will not be notably altered.

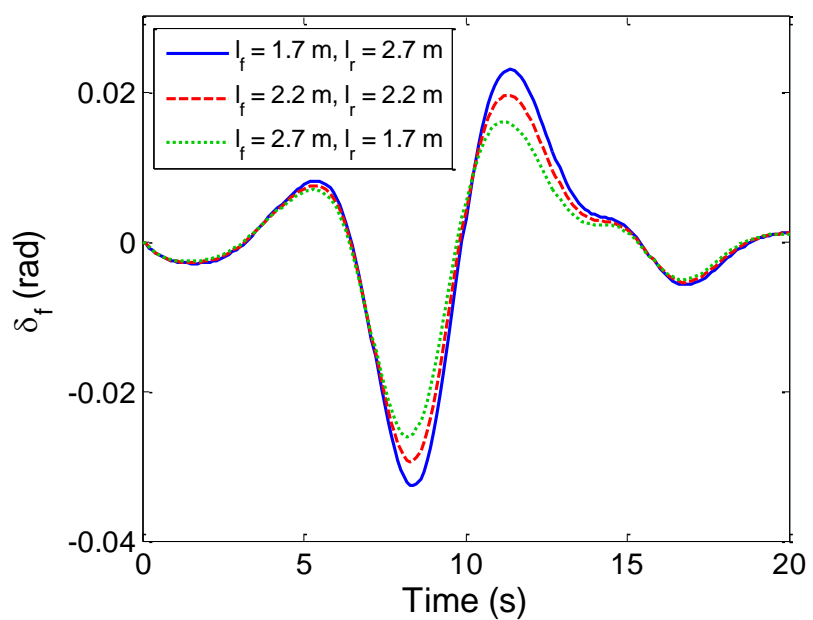

Fig.5. The estimated steering angles that vary with $l_{f}$ and $l_{r}$, while $l_{f}+l_{r}$ is kept constant $4.4 \mathrm{~m}$. The chosen trajectory is the no. 383 trajectory. The other parameters of the Bicycle model are chosen as $m=1500 \mathrm{~kg}, I_{z}=2500 \mathrm{kgm}^{2}, c_{f}=50000 \mathrm{~N} / \mathrm{rad}, c_{r}=64000 \mathrm{~N} / \mathrm{rad}$.

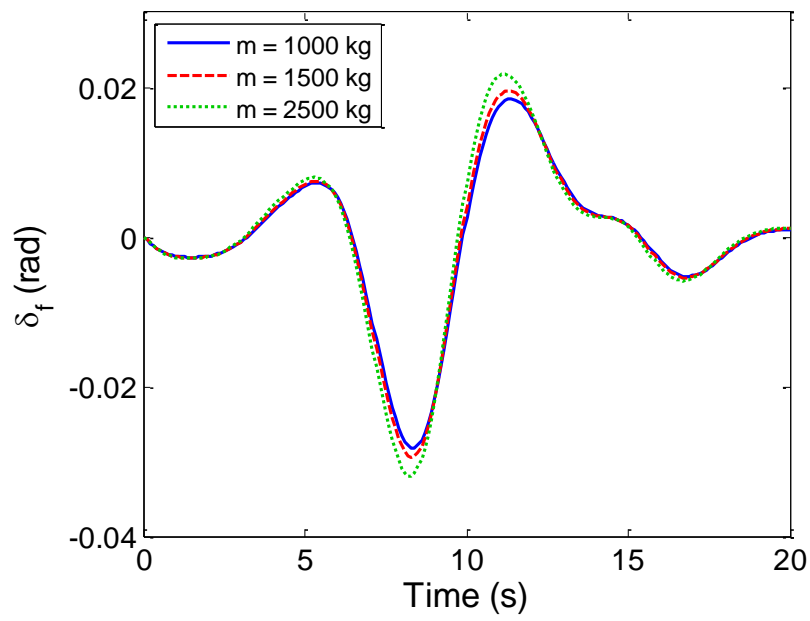

Fig.6. The estimated steering angles that vary with $m$ and $I_{z}$. The chosen trajectory is the no. 383 trajectory. The other parameters of the Bicycle model are chosen as $l_{f}=2.2 \mathrm{~m}, l_{r}=2.2 \mathrm{~m}, c_{f}=50000 \mathrm{~N} / \mathrm{rad}$,

$$
c_{r}=64000 \mathrm{~N} / \mathrm{rad}
$$

The above tests indicate that the estimation results are not sensitive to the uncertainties in length parameters $l_{f}$ and $l_{r}$, as well as mass parameter $m$ and $I_{z}$. Fig.7 shows the estimated steering angles times series when we vary $c_{f}$ as well as $c_{r}$ and keep other parameters unchanged. It indicates the estimated steering angles are sensitive to the uncertainties in stiffness coefficients. However, the steering angle time series estimated by using different $c_{f} / c_{r}$ have similar trends.

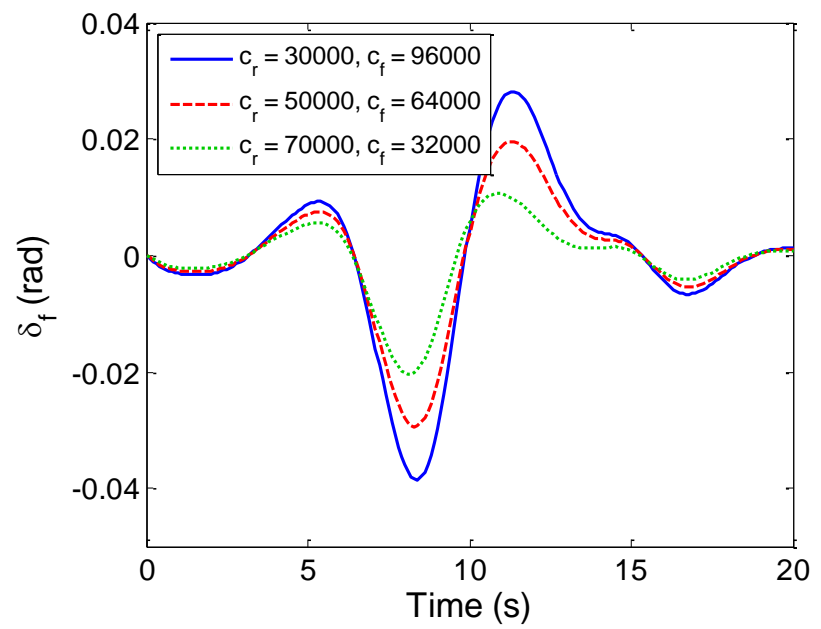

Fig.7. The estimated steering angles that vary with $m$ and $I_{z}$. The chosen trajectory is the no. 383 trajectory. The other parameters of the

Bicycle model are chosen as $l_{f}=2.2 \mathrm{~m}, l_{r}=2.2 \mathrm{~m}, m=1500 \mathrm{~kg}$,

$$
I_{z}=2500 \mathrm{kgm}^{2} \text {. }
$$

Since it is hard to quantitatively determine the uncertainty range of stiffness coefficients $c_{f}$ and $c_{r}$ from video data, we investigate why the varying trend of steering angle time series will be preserved no matter what values of $c_{f}$ and $c_{r}$ are used in Section III. The conclusion would help design better ADASs.

\section{The TARget HeAding ANGLE Model}

\section{A. Model Presentation}

Various models were proposed to mimic human's preview and predictive behavior during DLC. In this section, we will verify a simple target heading angle control model given in [34], based on the estimated steering angle time series.

The target point is defined as a look-ahead point away from current vehicle position. In [36], a driver was assumed to choose a point on the center line of adjacent lane (guideline in Fig.1) with a certain preview distance away as the target point, when he/she decides to make a lane change; see Fig.8. This target point was not fixed and would move forward along the center line of adjacent lane during the lane change. 


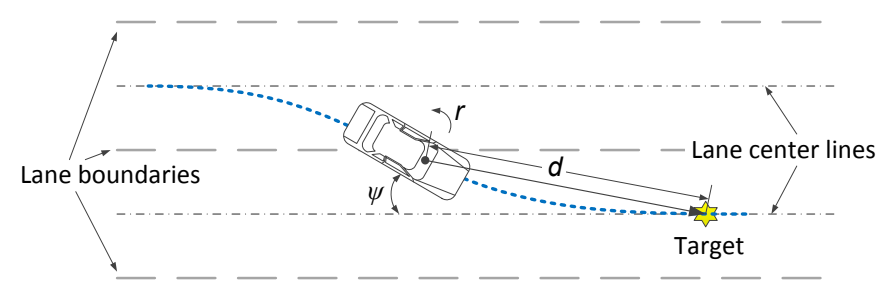

Fig. $\overline{8 .}$ An illustration for targeting point.

Suppose a drivers conducts steering prediction according to the so called target heading angle $\psi_{\text {des }}(t)$ which is the angle between the tangent line of vehicle trajectory and the guideline [36]. As shown in Fig.9. Let us define $P$ as current position of the CG of vehicle; $T$ as the target point; point $O$ as the center of the circle curve corresponding to the vehicle trajectory at time $t$, if the driver keeps its current yaw rate and speed; and $R(t)=O P=O T$ is the radius of this circle curve at time $t$. In this study, $R(t)$ is about $30 \mathrm{~m}$ and $l_{f}$ is about $2 \mathrm{~m}$. Since $R(t)$ is much larger than $l_{f}$, we directly take the displacement from $\mathrm{CG}$ to the guideline as $y_{f}$, without causing large error. We can then define $\alpha(t)$ to represent the angle between the $P T$ and $O P$, and $\theta(t)$ as the angle between guide line and $P T$. From Fig.9, we know that

$$
\begin{gathered}
\alpha(t)=\arccos \frac{d(t) / 2}{R(t)}=\arccos \frac{d(t) r(t)}{2 v(t)} \\
\theta(t)=\arcsin \frac{y_{f}(t)}{d}
\end{gathered}
$$

where $d$ is the preview distance.

Moreover, we have

$$
\psi_{\text {des }}(t)=\frac{\pi}{2}-\alpha(t)-\theta(t)=\arcsin \frac{d \cdot r(t)}{2 v(t)}-\arcsin \frac{y_{f}(t)}{d}
$$

holds for the heading angle.

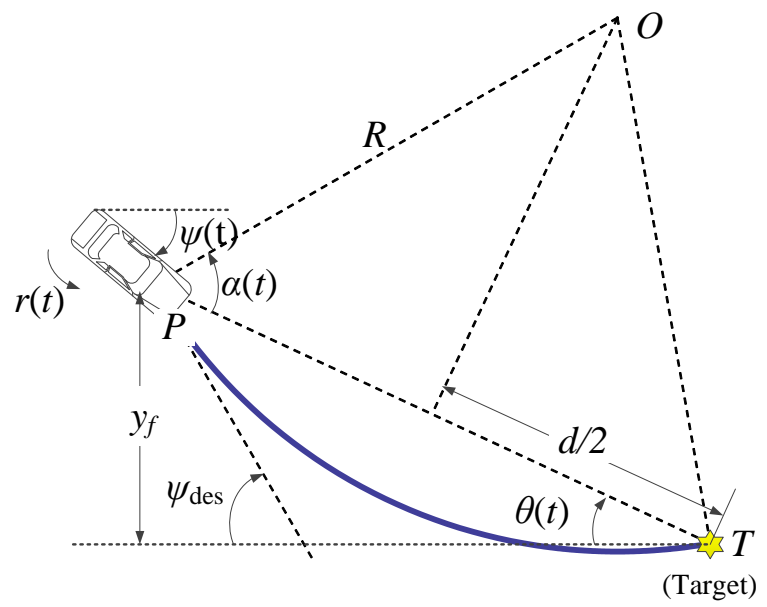

Fig.9. An illustration for targeting heading angle.

It was claimed in [36] that drivers do not consider a desired heading angle $\psi$ as a control command to turn the steering wheel. Instead, drivers consider the changing rate of heading angle as the reference. They will determine what the steering rate is needed based on the target angle error and turn the steering wheel accordingly.

To explain drivers' feedback on the target angle error, it was assumed in [36] that the steering rates are proportional to the heading angle errors

$$
\dot{\delta}_{f}(t)=k(t)\left[\psi_{\text {des }}(t-\Delta t)-\psi(t-\Delta t)\right]
$$

where $k(t)$ refers to the time-variant feedback gain, $\Delta t$ refers to the time delay between human eye perception and muscle response.

This steering law is in agreement with the model proposed in [46]. It captures the key steering mechanisms from a control perspective and meanwhile provides a psychophysical explanation for driver steering maneuvers. How to estimate $k(t)$ then becomes an important task, if we want to check the common steering characteristics shared by most drivers during DLC.

\section{B. Estimation of Control Law}

A direct estimation on $k(t)$ can be derived from the control law Eq.(7) as

$$
k(t)=\frac{\dot{\delta}_{f}(t)}{\psi_{\text {des }}(t-\Delta t)-\psi(t-\Delta t)}
$$

As mentioned in Section II.B, the steering rate $\dot{\delta}_{f}(t)$ and the heading angle $\psi(t)$ can be reconstructed from steering maneuvers algorithm proposed in previous section. If the preview distance is assumed to be a constant and is pre-selected, and the desired heading angle $\psi_{\text {des }}(t)$ is calculated via Eq.(6).

However, we need to determine the starting point of DLC maneuvers before calculating $k(t)$. As mentioned above, we had truncated all the studied trajectory segments by a 20 -second time window, but the actual duration for a DLC maneuver may be less than 20 seconds.

We had carefully investigated the bias of data error in reference and its influence on the duration of DLC actions [19]. Using the methods proposed in [19] together with smoothing techniques proposed in this paper, we could filter most influence of error data on DLC action modeling.

So, when we solved the inverse problem (3), the begin and end times of DLC actions can be also determined naturally, if we select a threshold for the steering angle $\delta_{f}(t)$ to check whether the DLC had happened. The threshold we set in this paper is the " $10 \%$ " rule.

A method proposed in [19] can be used to solve this problem, which is based on the lateral speed of vehicle. As shown in Fig.10, we start from time point of the max positive steering angle and search the first time point $T_{\text {end }}$ along the time axis when the value of steering angle falls below $10 \%$ of the max positive steering angle. Similarly, we start from time point of the max negative steering angle and search the time point $T_{\text {begin }}$ 
against the time axis when the value of steering angle rises above $10 \%$ of the max negative steering angle. The found $T_{\text {begin }}$ and $T_{\text {end }}$ are defined as the begin and end time points of this DLC maneuver.

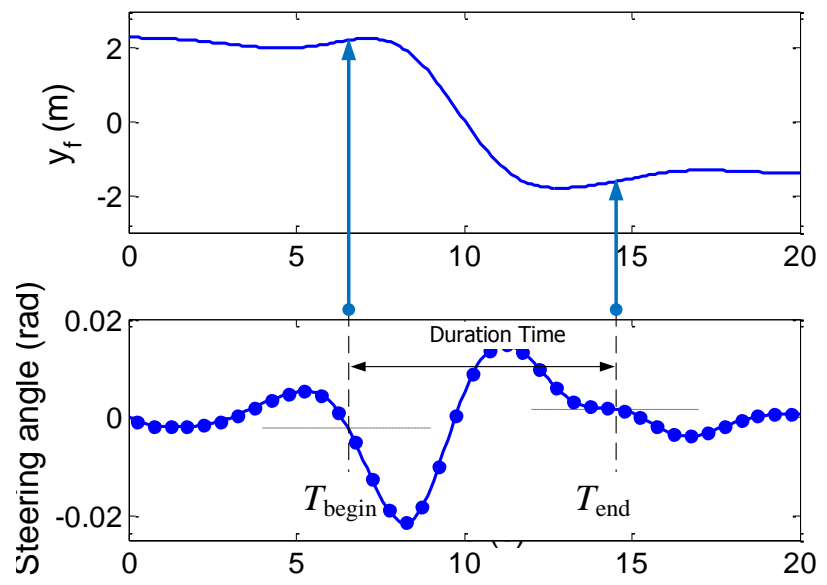

Fig.10. An illustration on how to determine the begin and end time points for a certain DLC action. The chosen trajectory is the no. 383 trajectory.

After determining the begin and end time points of a DLC maneuver, we can get the corresponding feedback gain from Eq.(8). Fig.11 shows $k(t)$ for the no. 383 trajectory. We can see that $k(t)$ contains two jerks, when the heading angle error is near zero. This phenomenon is caused by the numerical instability of Eq.(8) when the denominator is near zero.

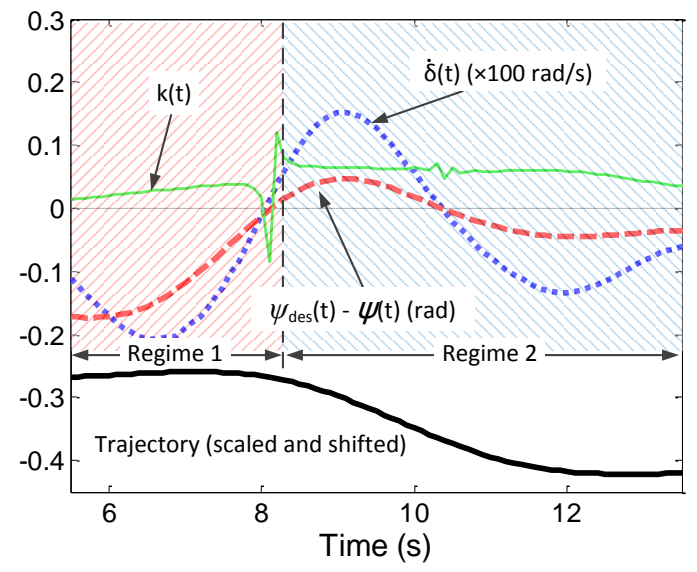

Fig.11. The estimated feedback gain $k(t)$ for the no. 383 trajectory, where we find $T_{\text {begin }}=7.5 \mathrm{~s}, T_{\text {end }}=12.2 \mathrm{~s}$ and $\Delta t=0.14 \mathrm{~s}$, after we calibrate the parameters from the trajectory.

Neglecting these two jerks, we can see that the entire process can be divided into two regimes. During the first regime (e.g. from $7.5 \mathrm{~s}$ to $12.2 \mathrm{~s}$ in Fig.11), the feedback gain increases gradually from 0 to a constant. During the second regime comes (e.g. from $8.5 \mathrm{~s}$ to $14 \mathrm{~s}$ in Fig.11), the feedback gain approximately keeps the same.
In order to find a minimum set to describe drivers' DLC maneuvers under different speed, we propose a four-factor set to characterize drivers' DLC maneuvers based on the above finding. These factors are:

(i) the preview distance $d$;

(ii) the increasing rate of $k(t)$ in the first regime;

(iii) the value of $k(t)$ in the second regime;

(iv) the perception-response delay $\Delta t$.

The last factor is considered, since several previous studies assumed that this factor may influence the preview control. As suggested in [36], the driver time delay is usually a small value between $0 \mathrm{~s}-0.2 \mathrm{~s}$.

To retrieve the driving features of massive drivers, we search a tetrad of these factors that produces a trajectory that fits the empirical trajectory best. More precisely, we get the feedback gain from Eq.(8) and calculate the corresponding steering actions $\delta_{f}(t)$. Then, we feed this $\delta_{f}(t)$ into Eq.(1) to get the simulated vehicle trajectory. Finally, we compare the simulated and empirical trajectories.

In this paper, we use the Root Mean Squared Error (RMSE) index to evaluate the difference between the model produced trajectory/steering angle and the empirical ones. Particularly, we consider the following four RMSE indices

$$
\begin{aligned}
& \operatorname{RMSE}_{1}=\sqrt{\frac{1}{T_{\text {end }}-T_{\text {start }} \int_{T_{\text {start }}}^{T_{\text {end }}}\left(y_{f}(t)-\hat{y}_{f}(t)\right)^{2} d t}} \\
& \mathrm{RMSE}_{2}=\sqrt{\frac{1}{T_{\text {end }}-T_{\text {start }}} \int_{T_{\text {start }}}^{T_{\text {end }}}\left(y_{f}^{\prime}(t)-\hat{y}_{f}^{\prime}(t)\right)^{2} d t} \\
& \mathrm{RMSE}_{3}=\sqrt{\frac{1}{T_{\text {end }}-T_{\text {start }}} \int_{T_{\text {start }}}^{T_{\text {end }}}\left(\delta_{f}(t)-\hat{\delta}_{f}(t)\right)^{2} d t} \\
& \mathrm{RMSE}_{4}=\sqrt{\frac{1}{T_{\text {end }}-T_{\text {start }} \int_{T_{\text {start }}}^{T_{\text {end }}}\left(\delta_{f}^{\prime}(t)-\hat{\delta}_{f}^{\prime}(t)\right)^{2} d t}}
\end{aligned}
$$

where $y_{f}(t)$ refers to the empirical trajectory and $\hat{y}_{f}(t)$ refers to the model produced one. $T_{\text {start }}$ and $T_{\text {end }}$ are the start and end time of a DLC process respectively. $y_{f}^{\prime}(t)$ and $\hat{y}_{f}^{\prime}(t)$ refers to the first order derivative of empirical trajectory and model produced one, respectively.

We add up these four RMSE values together to get the final evaluation index value. The smaller this value is, the better model produced trajectories fits empirical ones.

\section{THE COMmon Features of MASSIVE EXAMINEES}

Applying the method proposed in previous subsection, we checked all 1027 trajectories and estimated the corresponding four factors. The four factors in our model, namely gain value, gain increasing rate, delay, and preview distance, are plotted with respect to each vehicle's average speed during DLC, in bivariate scatter plot and marginal histogram plot; see Fig. 12-15.

The corresponding parameters of the bicycle model are chosen as $m=1500 \mathrm{~kg}, I_{z}=2500 \mathrm{~kg} \cdot \mathrm{m}^{2}, c_{f}=50000 \mathrm{~N} / \mathrm{rad}$, 
$c_{r}=64000 \mathrm{~N} / \mathrm{rad}, l_{f}=l_{r}=2.2 \mathrm{~m}$.

Based on the marginal histogram plots, it is clear that all these factors are neither uniformly nor normally distributed. However, the gain value, gain increasing rate and delay for driver do not change with respect to average speed during DLC; while preview distance changes roughly linearly with average speed. This indicates that the faster a driver runs a vehicle, the further target he/she will look at.

To quantitatively validate the possible dependences between these factors and speed, a dependence test is executed. Since these variables are neither uniformly nor normally distributed, the Hoeffding's test is the best way to evaluate their relations. Table II shows the corresponding results. The P-value indicates the critical value for rejecting the hypothesis that the two variables are independent at a confidence level of 0.05 . If the statistic result is less than the p-value, the hypothesis cannot be rejected and in other words, these two variables are dependent.

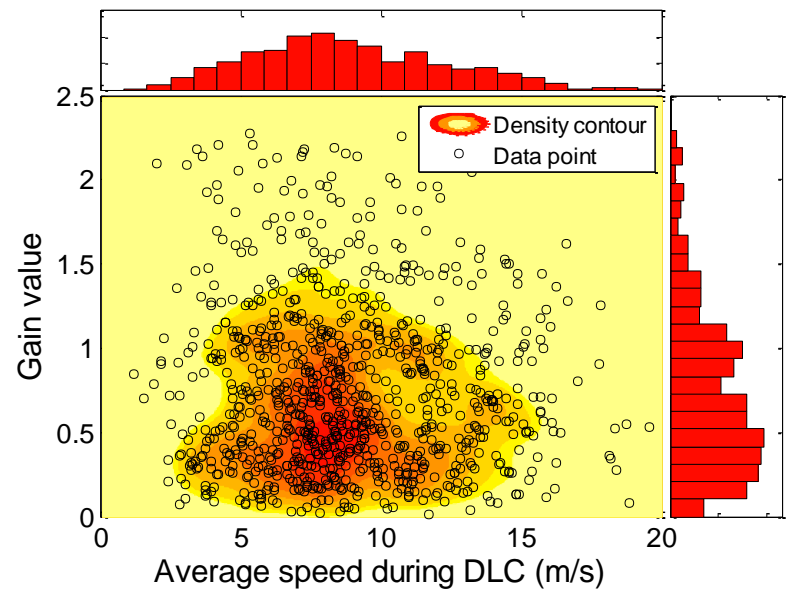

Fig 12. Scatter and histogram plot of gain value with respect to average speed. The contour plot clearly indicates that the distribution of gain value does not change with varies average speed.

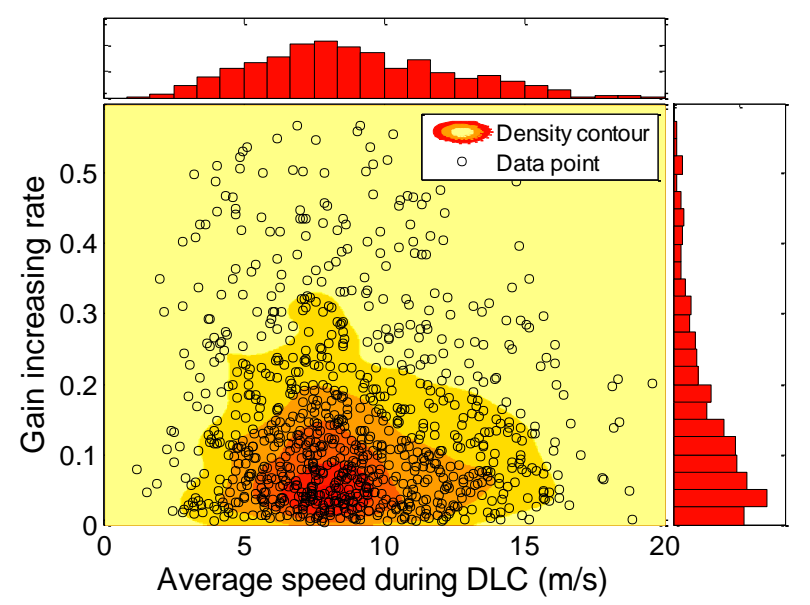

Fig 13. Scatter and histogram plot of gain increasing rate with respect to average speed.

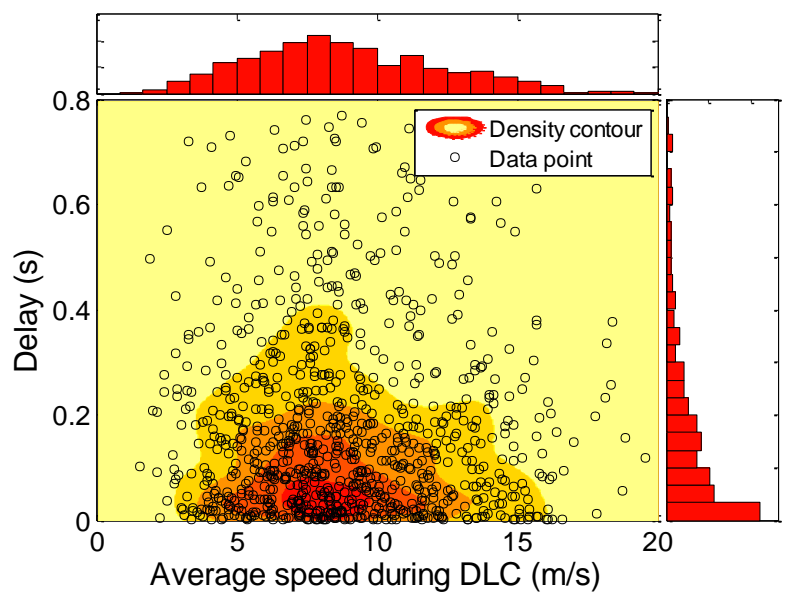

Fig 14. Scatter and histogram plot of delay with respect to average speed.

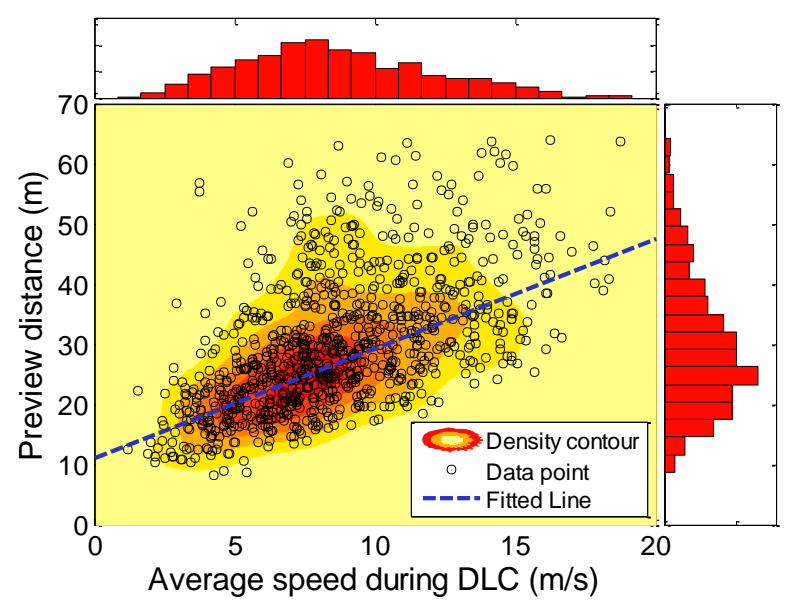

Fig 15. Scatter and histogram plot of preview distance with respect to average speed.

As expected, test results show that the first three factors, gain value, gain increasing rate, and delay, are independent with respect to speed; while, preview distance has a strong positive correlation with speed.

We estimated the relationship between preview distance and driving speed using a robust linear fit method proposed in [47]. This robust fit procedure uses reweighted least squares with a Talwar weighting function. The final fitted line is

$$
d(v)=11.07+1.82 v
$$

which indicates most drivers will look at a further target when they drive faster. Roughly, the preview distance will increase by $1.82 \mathrm{~m}$ when the speed increases by $1 \mathrm{~m} / \mathrm{s}$.

As mentioned in Section II.C, the major shortcoming of the proposed lane change behavior estimation method is we cannot accurately retrieve the stiffness coefficients $c_{f}$ and $c_{r}$ from video data. Thus, it is necessary to check whether the choice of $c_{f}$ and $c_{r}$ 's values will influence the above conclusions.

Further tests show that the joint distribution patterns of the factors and speed will not significantly change, if we choose different physical parameters of vehicles. Particularly, Fig.16 shows the relations between preview distance and speed, when 
three different parameter sets (shown in Table III) are adopted.

TABLE II

HOEFFDING'S TEST RESULT FOR FACTOR-SPEED PAIRS

\begin{tabular}{lccl}
\hline \hline \multicolumn{1}{c}{ Factor } & Statistic & P-Value & Conclusion \\
\hline $\begin{array}{l}\text { Gain } \\
\text { value }\end{array}$ & 0.000 & 0.606 & Do not reject \\
\hline $\begin{array}{l}\text { Gain } \\
\text { increasing } \\
\text { rate }\end{array}$ & 0.000 & 0.190 & Do not reject \\
\hline Delay & 0.000 & 0.321 & Do not reject \\
\hline $\begin{array}{l}\text { Preview } \\
\text { distance }\end{array}$ & 0.118 & 0.000 & Reject \\
\hline \hline
\end{tabular}

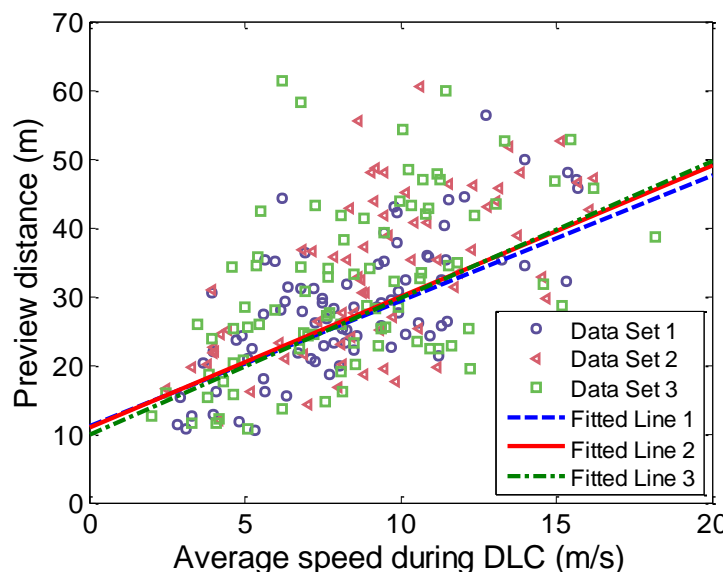

Fig 16. Scatter and histogram plot of preview distance with respect to average speed, where three different sets of vehicle parameters (shown in Table III) are adopted. To make the plot clear, we show only $15 \%$ points which are randomly sampled from each dataset.

TABLE III

DIFFERENT VEHICLE PARAMETER SETS

\begin{tabular}{lccc}
\hline \hline \multicolumn{1}{c}{ NAME } & SET1 & SET2 & SET3 \\
\hline$m(\mathrm{~kg})$ & 1500 & 2500 & 1000 \\
\hline$I_{z}\left(\mathrm{~kg} \cdot \mathrm{m}^{2}\right)$ & 2500 & 4167 & 1667 \\
\hline$c_{f}(\mathrm{~N} / \mathrm{rad})$ & 50000 & 30000 & 96000 \\
\hline$c_{f}(\mathrm{~N} / \mathrm{rad})$ & 64000 & 96000 & 30000 \\
\hline$l_{f}(\mathrm{~m})$ & 2.2 & 1.7 & 2.7 \\
\hline$l_{r}(\mathrm{~m})$ & 2.2 & 2.7 & 1.7 \\
\hline \hline
\end{tabular}

From Fig.16, we can see that the linear dependence between preview distance and speed remains almost unchanged. The other two fitted lines are

$$
\begin{gathered}
d(v)=11.00+1.90 v \\
d(v)=9.95+1.99 v
\end{gathered}
$$

Varying the parameters within the possible values, we find that the preview distance will usually increase by $1.8 \mathrm{~m} \sim 2.1 \mathrm{~m}$ when the speed increases by $1 \mathrm{~m} / \mathrm{s}$. This finding is in accordance with our daily driving experience and could be viewed as a common feature of drivers. The corresponding physiological factors behind this interesting phenomenon still needs further discussions.

\section{CONCLUSIONS}

In this paper, we propose a new method to retrieve common discretionary lane changing characteristics from lots of drivers, based on their recorded trajectories.

In short, we first explain how to solve the steering actions from the trajectories as an inverse problem, with the parameters of vehicle dynamics are assumed. Then, we assume that human drivers perceive future path information within a finite future distance through visual cues and apply a piecewise-linear feedback to control the angle between the preview target point and the road guideline. Test results support this assumption and further indicate that only preview distance changes roughly linearly with average speed during DLC.

However, it must be pointed out there are many alternative models in this field [48]-[49]. Constrained by the length limit, we only validate the estimated steering maneuvers with the model proposed in [36], [46]. We plan to check other models in our coming reports.

Besides, the recorded vehicle speeds are generally slow in the NGSIM data, since the data are collected at ramping region in rush hours and the traffic are congested. So, the DLC actions in high speed driving scenarios still need to be investigated in the future. We plan to collect more trajectory data in free flow states and study their features in the future.

This new method provides us a novel way to study drivers' normal behaviors outside the proving ground. We believe the obtained conclusions will benefit human behavior study, traffic safety analyze and traffic flow theory research. For example, based on the conclusions obtained, we may non-intrusively evaluate driving performance or physiological states of drivers based on online roadside traffic monitoring data (e.g. trajectory data collected from roadside video cameras). This opens a promising field of applications for enhancing driving safety under the newly emerging Cyber-Physics-Systems framework [50].

\section{APPENDIX I}

From the time-domain system dynamic equation shown in Eq.(1), we can get the following equations as shown in Eq. (A1)- Eq. (A4).

$$
\begin{array}{r}
\dot{\beta}(t)=a_{11} \beta(t)+a_{12} r(t)+b_{11} \delta_{f}(t) \\
\dot{r}(t)=a_{21} \beta(t)+a_{22} r(t)+b_{21} \delta_{f}(t) \\
\dot{\psi}(t)=r(t) \\
\dot{y}_{f}(t)=v \beta(t)+l_{f} r(t)+v \psi(t)
\end{array}
$$

Since we assume the longitudinal speeds of vehicles does not significantly change, we take $\dot{v} \approx \ddot{v} \approx 0$. So, $\ddot{y}_{f}(t)$ in the Appendix can be simplified as Eq. (A5), where $A_{11}, A_{12}, A_{13}$ are calculated in Eq. (A6)- Eq. (A8). 


$$
\begin{array}{r}
\ddot{y}_{f}(t)=A_{11} \beta(t)+A_{12} r(t)+A_{13} \delta_{f}(t) \\
A_{11}=v * a_{11}+l_{f} * a_{21} \\
A_{12}=v * a_{12}+l_{f} * a_{22}+v \\
A_{13}=v * b_{11}+l_{f} * b_{21}
\end{array}
$$

Further, we can calculate $\dddot{y}_{f}(t)$ based on $\ddot{y}_{f}(t)$, as shown in Eq. (A9), where $A_{21}, A_{22}, A_{23}, A_{24}$ are calculated in Eq. (A10)- Eq. (A13).

$$
\begin{aligned}
& \dddot{y}_{f}(t)= A_{21} \beta(t)+A_{22} r(t)+A_{23} \delta_{f}(t) \\
&+A_{24} \dot{\delta}_{f}(t) \\
& A_{21}=A_{11} * a_{11}+A_{12} * a_{21} \\
& A_{22}=A_{11} * a_{12}+A_{12} * a_{22} \\
& A_{23}=A_{11} * b_{11}+A_{12} * b_{21} \\
& A_{24}=A_{13}
\end{aligned}
$$

Further, we can calculate $y_{f}^{4}(t)$ based on $\dddot{y}_{f}(t)$, as shown in Eq. (A14), where $A_{31}, A_{32}, A_{33}, A_{34}, A_{35}$ are calculated in Eq. (A15)- Eq. (A19).

$$
\begin{array}{r}
y_{f}^{4}(t)=A_{31} \beta(t)+A_{32} r(t)+A_{33} \delta_{f}(t) \\
+A_{34} \dot{\delta}_{f}(t)+A_{35} \ddot{\delta}_{f}(t) \\
A_{31}=A_{21} * a_{11}+A_{22} * a_{21} \\
A_{32}=A_{21} * a_{12}+A_{22} * a_{22} \\
A_{33}=A_{21} * b_{11}+A_{22} * b_{21} \\
A_{34}=A_{23} \\
A_{35}=A_{24}
\end{array}
$$

Based on Eq. (A4), Eq. (A5), Eq. (A9) and Eq. (A14), we can construct a linear combination of $\dot{y}_{f}(t), \ddot{y}_{f}(t), \dddot{y}_{f}(t)$ and $y_{f}^{4}(t)$ to eliminate $\beta(t)$ and $r(t)$, as shown in Eq. (A20). $n_{1}, n_{2}, n_{3}, n_{4}, d_{0}, d_{1}$ and $d_{2}$ can be choose as shown in Eq. (A21)-Eq.(A27).

$$
\begin{gathered}
d_{0} \delta_{f}+d_{1} \dot{\delta}_{f}+d_{2} \ddot{\delta}_{f}=n_{1} \dot{y}_{f}+n_{2} \ddot{y}_{f}+n_{3} \dddot{y}_{f}+n_{4} y_{f}^{(4)} \\
n_{1}=0 \\
n_{2}=\frac{-A_{31} * A_{22}+A_{21} * A_{32}}{A_{11} * A_{22}-A_{12} * A_{21}} \\
n_{3}=\frac{-A_{11} * A_{32}+A_{12} * A_{31}}{A_{11} * A_{22}-A_{12} * A_{21}} \\
n_{4}=1 \\
d_{0}=n_{2} * A_{13}+n_{3} * A_{23}+1 * A_{33}
\end{gathered}
$$

$$
\begin{array}{r}
d_{1}=n_{3} * A_{24}+1 * A_{34} \\
d_{2}=1 * A_{35}
\end{array}
$$

\section{REFERENCES}

[1] M. C. Nechyba and Y. S Xu, "Stochastic similarity for validating human control strategy models," IEEE Transactions on Robotics and Automation, vol. 14, no. 3, pp. 437-451, 1998.

[2] J. H. Kim, S. Hayakawa, T. Suzuki, K. Hayashi, S. Okuma, N. Tsuchida, M. Shimizu, and S. Kido, "Modeling of driver's collision avoidance maneuver based on controller switching model," IEEE Transactions on Systems, Man, and Cybernetics-Part B: Cybernetics, vol. 35, no. 6, pp. 1131-1143, 2005.

[3] J. Wang, J. Wang, R. Wang, and C. Hu, "A Framework of Vehicle Trajectory Replanning in Lane Exchanging with Considerations of Driver Characteristics," IEEE Transactions on Vehicular Technology, 2016, in press. http://ieeexplore.ieee.org/document/7565531/

[4] V. A. Butakov, and P. Ioannou. "Personalized driver/vehicle lane change models for ADAS." IEEE Transactions on Vehicular Technology, vol. 64, no.10, pp. 4422-4431, 2015.

[5] Hou, Y., Zhao, Y., Wagh, A., et al., Simulation-Based Testing and Evaluation Tools for Transportation Cyber-Physical Systems. IEEE Transactions on Vehicular Technology, vol. 65, no. 3, pp.1098-1108, 2016.

[6] X. Zhu, H. Zhang, J. Wang, Z. Fang, "Robust Lateral Motion Control of Electric Ground Vehicles With Random Network-Induced Delays," IEEE Transactions on Vehicular Technology, vol. 64, no.11, 4985-4995, 2015.

[7] L. Li, D. Wen, N.-N. Zheng, and L.-C. Shen, "Cognitive cars: A new frontier for ADAS research," IEEE Transactions on Intelligent Transportation Systems, vol. 13, no. 1, pp. 395-407, 2012.

[8] M. Rahman, M. Chowdhury, Y. Xie, and Y. He, "Review of microscopic lane-changing models and future research opportunities," IEEE Transactions on Intelligent Transportation Systems, vol. 14, no. 4, pp. 1942-1956, 2013.

[9] C. F. Choudhury, Modeling Driving Decisions with Latent Plans, Ph.D. Dissertation, MIT, Cambridge, MA, 2007.

[10] S. Schnelle, J. Wang, H. Su, and R. Jagacinski, “A Personalizable Driver Steering Model Capable of Predicting Driver Behaviors in Vehicle Collision Avoidance Maneuvers," IEEE Transactions on Human-Machine Systems, 2016, in press. http://ieeexplore.ieee.org/document/7581066/

[11] S. Khosravani, A. Kasaiezadeh, A. Khajepour, B. Fidan, S.K. Chen, B. Litkouhi, " Torque-Vectoring-Based Vehicle Control Robust to Driver Uncertainties," IEEE Transactions on Vehicular Technology, vol. 64, no. 8, pp. 3359-3367, 2015.

[12] J. Wang, G. Zhang, R. Wang, S. Schnelle, J. Wang, "A Gain-Scheduling Driver Assistance Trajectory Following Algorithm Considering Different Driver Steering Characteristics," IEEE Transactions on Intelligent Transportation Systems , 2016, in press. http://ieeexplore.ieee.org/document/7548349/

[13] R. A. Hess and A. Modjtahedzadeh, "A control theoretic model of driver steering behavior," IEEE Control Systems Magazine, vol. 10, no. 5, pp. 3-8, 1977.

[14] L.-K. Chen and A. G. Ulsoy, "Identification of a driver steering model, and model uncertainty, from driving simulator data," ASME Journal of Dynamic Systems, Measurement, and Control, vol. 123, vo. 4, pp. 623-629, 2001.

[15] M. L. Ho, P. T. Chan, and A. B. Rad, "Lane change algorithm for autonomous vehicles via virtual curvature method," Journal of Advanced Transportation, vol. 43, no. 1, pp. 47-70, 2009.

[16] F.-Y. Wang, X. Wang, L. Li, and P. Mirchandani, "Creating a digital-vehicle proving ground," IEEE Intelligent Systems Magazine, vol. 18 , no. 2, pp. 12-15, 2003.

[17] X. Li, Z. Sun, Z., D. Cao, D. Liu, H. He, "Development of a new integrated local trajectory planning and tracking control framework for autonomous ground vehicles," Mechanical Systems and Signal Processing, vol. 87, Part B, pp. 118-137, 2017.

[18] Y. Xuan and B. Coifman, "Identifying lane-change maneuvers with probe vehicle data and an observed asymmetry in driver accommodation," ASCE Journal of Transportation Engineering, vol. 138, no. 8, pp. 1051-1061, 2012 
[19] Q. Wang, Z. Li, and L. Li, "Investigation of discretionary lane change characteristics using NGSIM datasets," Journal of Intelligent Transportation Systems: Technology, Planning, and Operations, vol. 18, no. 3, pp. 246-253, 2014.

[20] Z. Kim and J. Malik, "High-quality vehicle trajectory generation from video data based on vehicle detection and description," Proceedings of IEEE Conference on Intelligent Transportation Systems, pp. 176-182, 2003.

[21] K. Wang, W. Huang, B. Tian, and D. Wen, "Measuring driving behaviors from live video," IEEE Intelligent Systems, vol. 27, no. 5, pp. 75-80, 2012.

[22] The Next Generation Simulation (NGSIM) program, http://ngsim-community.org/

[23] L. Li and F.-Y. Wang, "Parking guidance system for front wheel steering vehicles using trajectory generation," Proceedings of IEEE Conference on Intelligent Transportation Systems, pp. 1770-1775, 2003.

[24] L. Li and F.-Y. Wang, Advanced Motion Control and Sensing for Intelligent Vehicles, Springer, New York, 2007.

[25] X. Li, Z. Sun, Z., D. Cao, D., Z. He, Z., Q. Zhu, "Real-Time Trajectory Planning for Autonomous Urban Driving: Framework, Algorithms, and Verifications," IEEE/ASME Transactions on Mechatronics, vol.21, no.2, 740-753, 2015.

[26] G. Xu, L. Liu, Y. Ou, and Z. Song, "Dynamic modeling of driver control strategy of lane-change behavior and trajectory planning for collision prediction," IEEE Transactions on Intelligent Transportation Systems, vol. 13, no. 3, pp. 1138-1155, 2012.

[27] Y. Xu, J. Song, M. C. Nechyba, and Y. Yam, "Performance evaluation and optimization of human control strategy," Robotics and Autonomous Systems, vol. 39, no. 1, pp. 19-36, 2002.

[28] T. Limpibunterng and T. Fujioka, "Bilateral driver model for steer-by-wire controller design," Vehicle System Dynamics Supplement, vol. 41, pp. 381-390, 2004.

[29] K. Guo, Y. Cheng, and H. Ding, "Analytical method for modeling driver in vehicle directional control," Vehicle System Dynamics Supplement, vol. 41, pp. 401-410, 2004.

[30] C. Lv, Y. Liu, X. Hu, H. Guo, D. Cao, F.-Y. Wang, "Simultaneous Observation of Hybrid States for Cyber-Physical Systems: A Case Study of Electric Vehicle Powertrain," IEEE Transactions on Cybernetics, 2017.

[31] R. Hayama, S. Kawahara, S. Nakano, and H. Kumamoto, "Resistance torque control for steer-by-wire system to improve human machine interface," Vehicle System Dynamics, vol. 48, no. 9, pp. 1065-1075, 2010.

[32] C. C. MacAdam, "Application of an optimal preview control for simulation of closed-loop automobile driving," IEEE Transactions on Systems, Man, and Cybernetics Part C, vol. 11, no. 6, pp. 393-399, 1981.

[33] A. M. C. Odhams and D. J. Cole, "Application of linear preview control to modelling human steering control," Proceedings of the Institution of Mechanical Engineers, Part D: Journal of Automobile Engineering, vol. 223, no. 7, pp. 835-853, 2009.

[34] S. D. Keena and D. J. Cole, "Application of time-variant predictive control to modelling driver steering skill," Vehicle System Dynamics, vol. 49, no. 4, pp. 527-559, 2011.

[35] S. D. Keen and D. J. Cole, "Bias-Free identification of a linear modelpredictive steering controller from measured driver steering behavior," IEEE Transactions on Systems, Man, and Cybernetics-Part B: Cybernetics, vol. 42, no. 2, pp. 434-443, 2012.

[36] H. S. Tan and J. H. Huang, "Experimental development of a new target and control driver steering model based on DLC test data," IEEE Transactions on Intelligent Transportation Systems, vol. 13, no. 1, pp. 375-384, 2012.

[37] Q. Wang, J. Zhang, L. Li, and J. Hu, "A control model for human steering actions during lane change based on NGSIM dataset," Proceedings of IEEE Conference on Intelligent Transportation Systems, pp. 359-364, 2011.

[38] C. Lv, H. Wang, H., and D. Cao, "High-Precision Hydraulic Pressure Control Based on Linear Pressure-Drop Modulation in Valve Critical Equilibrium State, " IEEE Transactions on Industrial Electronics, 2017.

[39] L. Li, F.-Y. Wang, and Q. Zhou, "Integrated longitudinal and lateral tire/ road friction modeling and monitoring for vehicle motion control," IEEE Transactions on Intelligent Transportation Systems, vol. 7, no. 1, pp. 1-19, 2006.

[40] Z. Fu, W. Hu, T. Tan, "Similarity based vehicle trajectory clustering and anomaly detection," Proceedings of IEEE International Conference on Image Processing, no. II 602-5, 2005.
[41] S. Atev, G. Miller, and N. P. Papanikolopoulos, "Clustering of vehicle trajectories," IEEE Transactions on Intelligent Transportation Systems, vol. 11, no. 3, pp. 647-657, 2010.

[42] H. Prautzsch, W. Boehm, M. Paluszny, Bézier and B-Spline Techniques, Springer, 2002.

[43] J. D. Lambert, Numerical Methods for Ordinary Differential Systems, Wiley, New York, 1992.

[44] D. D. Salvucci and A. Liu, "The time course of a lane change: Driver control and eye-movement behavior," Transportation Research Part F: Traffic Psychology and Behaviour, vol. 5, no. 2, pp. 123-132, 2002.

[45] T. Toledo and D. Zohar, "Modeling duration of lane changes," Transportation Research Record, no. 1999, pp. 71-78, 2007.

[46] T. Gordon and N. Magnuski, "Modeling normal driving as a collision avoidance process," Proceedings of International Symposium on Advanced Vehicle Control, 2006.

[47] D. P. O'Leary, "Robust regression computation using iteratively reweighted least squares," SIAM Journal on Matrix Analysis and Applications, vol. 11, no. 3, pp. 466-480, 1990.

[48] G. Markkula, "A review of near-collision driver behavior models," Human Factors, vol. 54 no. 6, pp. 1117-1143, December 2012.

[49] G. Markkula, O. Benderius, and M. Wahde, "Comparing and validating models of driver steering behaviour in collision avoidance and vehicle stabilisation," Vehicle System Dynamics, vol. 52, no. 12, pp. 1658-1680, 2014.

[50] F.-Y. Wang, "The emergence of intelligent enterprises: From CPS to CPSS," IEEE Intelligent Systems, vol. 25, no. 4, pp. 85-88, 2010. 
2017-11-09

Retrieving common discretionary lane changing characteristics from trajectories

$\mathrm{Li}, \mathrm{Li}$

IEEE

Li L, Lv C, Cao D, Zhang J, Retrieving common discretionary lane changing characteristics from trajectories, IEEE Transactions on Vehicular Technology, Volume: 67, Issue: 3, March 2018, pp2014-2024 http://dx.doi.org/10.1109/TVT.2017.2771144

Downloaded from Cranfield Library Services E-Repository 